\title{
'||||||||||||||||||||||||||||||||||||||||||||||||||||||||||||||||||||.
}

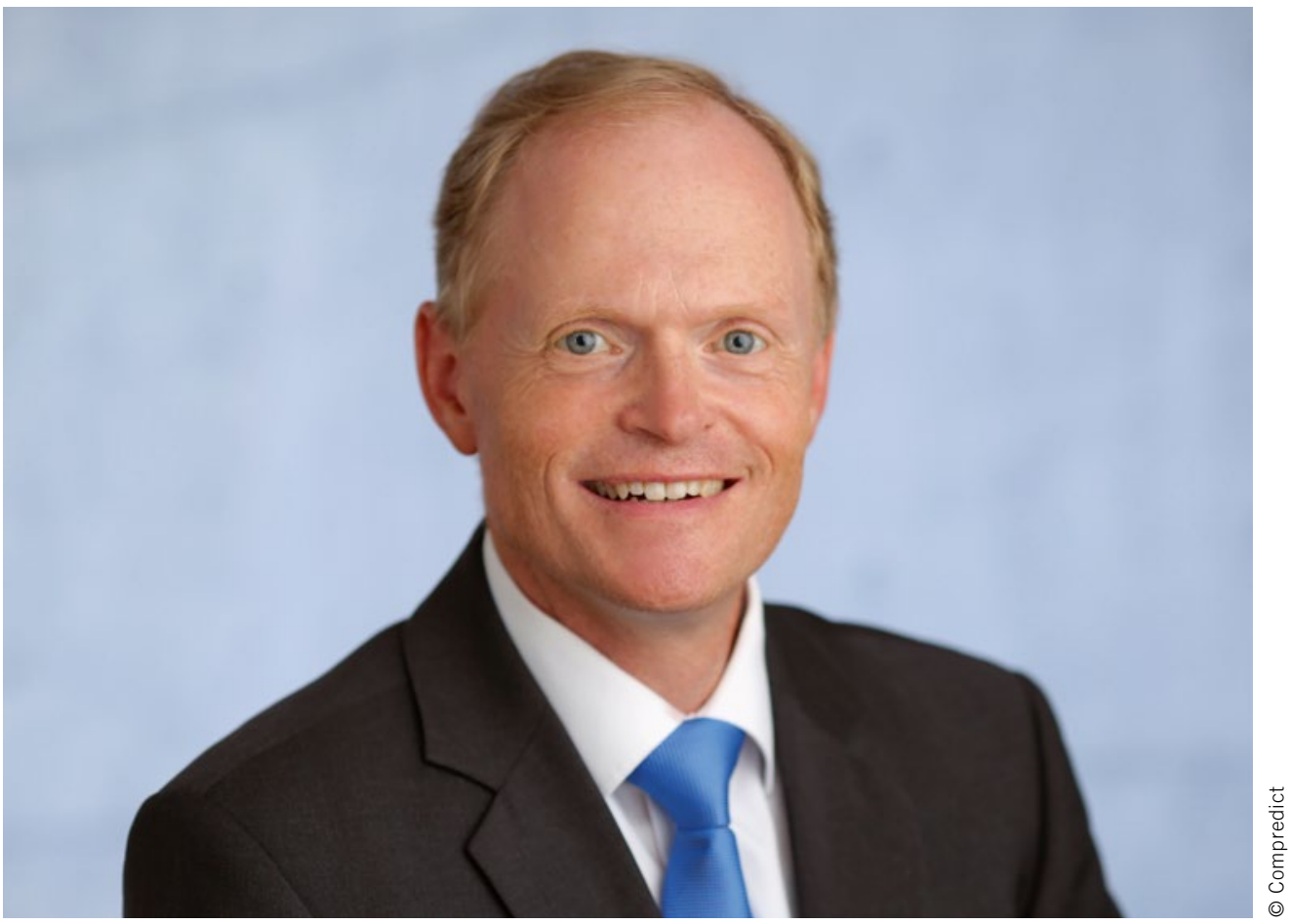

Prof. Dr.-Ing.

Stephan Rinderknecht

Director of the Institute

for Mechatronic Systems

in Mechanical Engineering

at the Technische Universität

(TU) Darmstadt (Germany)

\section{Overall System - The Key to Electric Mobility}

Many people currently regard range, cost, and charging infrastructure as the major challenges facing e-vehicles. Experts and the public agree that electric cars will become widespread on our roads, but the question is when this will happen. Opinions vary from electric cars making up the majority of new registrations in just a few years' time through to major market penetration only after a few decades have passed. While one side is focusing on protecting the environment and rapidly replacing outdated technologies, the other believes in using the existing potential of combustion engines to provide future mobility solutions that make financial and environmental sense.

Neither of these polarized viewpoints does justice to the problem and therefore a different approach is needed. The following questions are an example of this: Should the $\mathrm{CO}_{2}$ emissions of an electric car be measured on the basis of the electricity mix or should the additional $\mathrm{CO}_{2}$ emitted as a result of the actual increase in electricity consumption be estimated in the power grid? Is the assessment of the energy needed taken from the standard driving cycles or is there an environmental assessment available that includes the provision of the energy, the manufacture and disposal of the vehicles based on real usage profiles?

It is clear that electric cars will lead to an increase in demand for electricity. However, the energy transition requires a reduction in electricity consumption so that the proportion of renewable energy being used can be increased in accordance with the government's plans. The result is two conflicting objectives that can only be achieved by an integrated approach to energy generation from the perspective of the overall system.

It is well known that there are notable differences between the actual fuel consumption of cars and the figures provided by standard driving cycle tests and that this discrepancy is even emphasized by e-vehicles. For example, interior heating of conventional cars has almost no impact on fuel consumption as the heat is produced as an integral part of the system. But only costly electric heating is available in e-vehicles. The new WLTP driving cycle does not realistically reflect this. A research project at the TU Darmstadt entitled "Knowledge-based car 5.0" is currently investigating an innovative big data process for synthesizing driving cycles that have been improved to represent real-life driving conditions. The process allows actual usage profiles, including the ambient temperature, to be recorded.

There are many challenges involved in the widespread introduction of electric cars and it is essential for the energy transition and the actual electricity consumption to be taken into account. The key lies in a more comprehensive evaluation of the overall system. There are a number of factors in favor of ongoing moderate change. Vehicles with zero local emissions will be essential in our cities and technical competition on the market will determine whether cars covering longer distances should be powered by batteries or $\mathrm{CO}_{2}$-neutral range extenders. 